\title{
AKT serine/threonine protein kinase modulates baicalin- triggered autophagy in human bladder cancer $\mathrm{T} 24$ cells
}

\author{
CHINGJU LIN ${ }^{1}$, SHIH-CHANG TSAI ${ }^{2}$, MICHAEL T. TSENG ${ }^{10}$, SHU-FEN PENG ${ }^{2}$, SHENG-CHU KUO ${ }^{6,7}$, \\ MENG-WEI LIN ${ }^{8}$, YUAN-MAN HSU ${ }^{2}$, MIAU-RONG LEE ${ }^{5}$, SAKAE AMAGAYA ${ }^{11}$, \\ WEN-WEN HUANG ${ }^{2}$, TIAN-SHUNG WU ${ }^{3,6,9}$ and JAI-SING YANG ${ }^{4}$ \\ Departments of ${ }^{1}$ Physiology, ${ }^{2}$ Biological Science and Technology, ${ }^{3}$ Pharmacy, ${ }^{4}$ Pharmacology and ${ }^{5}$ Biochemistry, \\ ${ }^{6}$ Graduate Institute of Pharmaceutical Chemistry, China Medical University, Taichung 404; \\ ${ }^{7}$ Chinese Medicine Research and Development Center, China Medical University Hospital, Taichung 401; \\ ${ }^{8}$ Department of Nursing, Cardinal Tien College of Healthcare and Management, Taipei 23143; ${ }^{9}$ Department of Chemistry, \\ National Cheng Kung University, Tainan 701, Taiwan, R.O.C.; ${ }^{10}$ Department of Anatomical Sciences \\ and Neurobiology, School of Medicine, University of Louisville, KY 40202, USA; \\ ${ }^{11}$ Department of Kampo Pharmaceutical Sciences, Nihon Pharmaceutical University, Saitama 362-0806, Japan
}

Received November 8, 2012; Accepted December 28, 2012

DOI: $10.3892 /$ ijo.2013.1791

\begin{abstract}
Baicalin is one of the major compounds in the traditional Chinese medicinal herb from Scutellaria baicalensis Georgi. We investigated the molecular mechanisms of cell autophagy induced by baicalin in human bladder cancer T24 cells. Baicalin inhibited cell survival as shown by MTT assay and increased cell death by trypan blue exclusion assay in a concentration-dependent manner. Baicalin did not induce apoptotic cell death in T24 cells by TUNEL and caspase-3 activity assay. Baicalin induced the acidic vesicular organelle cell autophagy marker, manifested by acridine orange (AO) and monodansylcadaverine (MDC) staining and cleavage of microtubule-associated protein 1 light chain 3 (LC3). The protein expression levels of the Atg 5, Atg 7, Atg 12, Beclin-1 and LC3-II were upregulated in T24 cells after baicalin treatment. Inhibition of autophagy by 3-methyl-adenine (an inhibitor of class III phosphatidylinositol-3 kinase; 3-MA) reduced the cleavage of LC3 in T24 cells after baicalin treatment. Furthermore, protein expression levels of phospho-AKT (Ser473) and enzyme activity of AKT were downregulated
\end{abstract}

Correspondence to: Dr Jai-Sing Yang, Department of Pharmacology, China Medical University, No. 91 Hsueh-Shih Road, Taichung 40402, Taiwan, R.O.C.

E-mail: jaising@mail.cmu.edu.tw

Professor Tian-Shung Wu, Department of Chemistry, National Cheng Kung University, 1 Ta-Hsueh Road, Tainan 70101, Taiwan, R.O.C.

E-mail: tswu@mail.ncku.edu.tw

E-mail: tswu@mail.cmu.edu.tw

Key words: baicalin, autophagy, AKT, traditional Chinese medicine, T24 cells in T24 cells after baicalin treatment. In conclusion, baicalin triggered cell autophagy through the AKT signaling pathway in T24 cells.

\section{Introduction}

The morphological processes leading to cell death include apoptosis, necrosis and autophagy (1-3). Autophagy (or called self-eating) is a process maintaining cellular homeostasis $(4,5)$. When the cells undergo cellular damage, autophagy is required for the promotion of cellular survival $(4,6)$. Autophagy involves the autophagosome formation (a doublemembrane structure), which fuses with a lysosome to form an autophagolysosome, finally resulting in degradation of the captured proteins or organelles by lysosomal enzymes $(7,8)$. Several reports have shown that autophagy-related (Atg) proteins and microtubule-associated protein 1 light chain 3 (LC3) are major proteins involved in autophagy processes (9-11). Induction of Atg and LC3 protein levels has been linked with altering a variety of cellular signaling pathways, such as adenosine monophosphate-activated protein kinase (AMPK) (12-14), mitogen-activated protein kinase (MAPK) $(15,16)$ and PI3K/Akt pathways $(17,18)$. Previous studies indicated that suppression of PI3K/Akt is involved in regulating autophagy formation (19-21).

Chinese herbs are used for treatment of diseases in Taiwan and in China for a long time $(22,23)$. Baicalin is one of the major flavonoids (molecular formula: C21H18O11; Fig. 1) in the traditional Chinese medicinal herb 'Huang qin' (Scutellaria baicalensis Georgi) (24,25). The baicalin exhibits many different pharmacological actions such as anti-oxidant (26), photo-protective (27), neural protective $(28,29)$, antidepressant (30), anti-inflammatory $(31,32)$, anti-viral $(33,34)$, anti-hepatotoxicity $(35,36)$ and anticancer effects (37-39). Baicalin induces CA46 Burkitt lymphoma cell apoptosis through inhibiting the PI3K/Akt kinase activity (40). Baicalin 
induces apoptosis in SW620 colorectal cancer cells in vitro and anticancer activity in HCT-116 cells in vivo $(41,42)$, and Zheng et al demonstrated that baicalin induces apoptosis in leukemia HL-60/ADR cells through inhibiting the PI3K/Akt kinase (43). Our previous study demonstrated that baicalin induced apoptosis in leukemia HL-60 cells through ER stress and mitochondrial-dependent pathways (44). Recently, Zhang et al pointed out that baicalin induces autophagy in human hepatocellular carcinoma SMMC-7721 cells (45); however, there is no evidence to show the effects of baicalin on the induction of autophagy in human bladder cancer T24 cells. In the present study, we investigated the pharmacological effects of the baicalin on inhibition of cell growth and induction of cell autophagy in T24 cells. Our results indicated that baicalin might contribute to cell autophagy via the Akt pathway in T24 cells.

\section{Materials and methods}

Chemicals and reagents. Acridine orange (AO), Baicalin, 3methyladenine (3-MA), monodansylcadaverine (MDC) and tetrazolium 3-(4,5-dimethylthiazol-2-yl)-2,5-diphenyltetrazolium bromide (MTT) were purchased from Sigma-Aldrich Corp. (St. Louis, MO, USA). Fetal bovine serum (FBS), L-glutamine, penicillin/streptomycin and trypsin-EDTA were obtained from Invitrogen Life Technologies (Carlsbad, CA, USA). AKT kinase assay kit was obtained from Cell Signaling Technology (Danvers, MA, USA). Tdt-mediated deoxyuridine triphosphate nick end labeling (TUNEL) assay kit was purchased from Roche Diagnostics (GmBH, Mannheim, Germany). Caspase-3 activity assay kit was purchased from R\&D Systems Inc. (Minneapolis, MN, USA). The primary antibodies against Atg 5, Atg 7 and Atg 12, Beclin, LC3-II, AKT and phospho-AKT (Ser473) were purchased from Cell Signaling Technology. Antibody against $\beta$-actin was obtained from Sigma Chemical Co. All peroxidase-conjugated secondary antibodies were obtained from Santa Cruz Biotechnology Inc. (Santa Cruz, CA, USA). The enhanced chemiluminescence (ECL) detection kit was obtained from Pierce Chemical (Rockford, IL, USA).

Cell culture. The T24 human bladder cancer cell line was purchased from the Food Industry Research and Development Institute (Hsinchu, Taiwan). The cells were grown in McCoy's 5a medium fortified with $10 \%$ FBS, 2 mM L-glutamine and penicillin/streptomycin and incubated at $37^{\circ} \mathrm{C}$ under a humidified $5 \% \mathrm{CO}_{2}$ atmosphere (46).

Cell viability and morphology. The cell viability was assessed by the MTT assay. Briefly, the T24 cells were cultured in a 96-well plate at the density of $1 \times 10^{4}$ cells/per well and were incubated with $0,50,100,150$ and $200 \mu \mathrm{M}$ of baicalin for $24 \mathrm{~h}$. For the study of autophagy inhibition, cells were pre-treated with 3-MA $(10 \mathrm{mM})$ for $1 \mathrm{~h}$ before the 24 -h treatment of baicalin with indicated concentrations. At the end of baicalin treatment, culture medium containing MTT $(0.5 \mathrm{mg} / \mathrm{ml})$ was added to each well after washing the cells. The cells were then incubated at $37^{\circ} \mathrm{C}$ for $4 \mathrm{~h}$ and the supernatant was removed. The formed blue formazan crystals in viable T24 cells were dissolved with isopropanol/ $/ 0.04 \mathrm{~N} \mathrm{HCl}$, followed by measure-

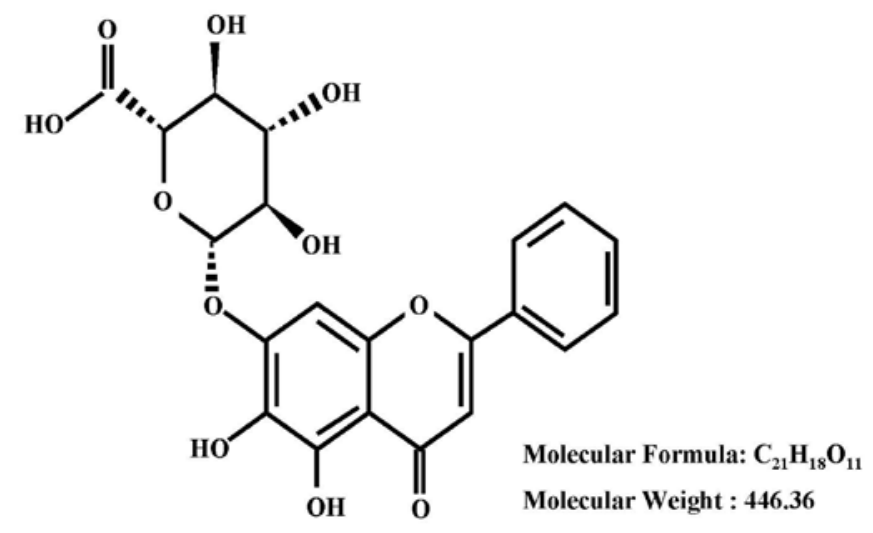

$\underline{\text { Baicalin }}$

Figure 1. The chemical structure of baicalin.

ment of the absorbance of each well at $570 \mathrm{~nm}$ with the ELISA reader with a reference wavelength of $620 \mathrm{~nm}$. All experiments were performed in triplicate. The cell viability of each treatment was expressed as percentage of the control. The morphological examination of autophagic vacuoles in baicalin-treated cells was determined under a phase-contrast microscope (18).

Trypan blue exclusion assay for cell death. Trypan blue exclusion assay was used to evaluate cell death induced by baicalin treatment. T24 cells in a 24 -well plate $\left(2.5 \times 10^{5}\right.$ cells/per well $)$ were incubated with $0,50,100,150$ and $200 \mu \mathrm{M}$ of baicalin. After $24 \mathrm{~h}$, cells were stained with $0.25 \%$ trypan blue solution and the numbers of dead cells were determined by Countess Automated Cell Counter (Invitrogen/Life Technologies) (18).

TUNEL staining. TUNEL staining was performed to detect apoptotic cells according to the manufacturer's protocol (in situ cell death detection kit; Roche Diagnostics). T24 cells in a 24 -well plate $\left(2.5 \times 10^{5}\right.$ cells/per well) were exposed to 0 , $50,100,150$ and $200 \mu \mathrm{M}$ of baicalin for $24 \mathrm{~h}$. At the end of the incubation, cells were collected, fixed with $70 \%$ ethanol and washed twice with ice-cold PSB. After incubated in the dark for $30 \mathrm{~min}$ at $37^{\circ} \mathrm{C}$ in $100 \mu \mathrm{l}$ of TdT-containing solution, the T24 samples were washed once before flow cytometry analysis of the TUNEL-positive cells using a FACSCalibur (BectonDickinson). The median fluorescence intensity was quantified by CellQuest software (18).

Caspase- 3 activity assays. The caspase- 3 activity assay was performed according to the manufacturer's instructions (Caspase Colorimetric Kit; R\&D Systems Inc.). Briefly, after a 24-h incubation with $0,50,100,150$ and $200 \mu \mathrm{M}$ of baicalin, T24 cells ( $1 \times 10^{7} / 75-\mathrm{T}$ flask) were harvested. The collected cells were then lysed in the lysis buffer $[50 \mathrm{mM}$ Tris- $\mathrm{HCl}(\mathrm{pH}$ 7.4), $1 \mathrm{mM}$ EDTA, $10 \mathrm{mM}$ EGTA, $10 \mathrm{mM}$ digitonin and $2 \mathrm{mM}$ DTT], followed by centrifugation to collect total proteins in the supernatant. The cell lysate containing $50 \mu \mathrm{g}$ proteins were then incubated for $1 \mathrm{~h}$ at $37^{\circ} \mathrm{C}$ with caspase- 3 specific substrate (Ac-DEVD-pNA) in the reaction buffer. The caspase- 3 activity was determined by measuring $\mathrm{O}_{\mathrm{D} 405}$ of the released pNA (18). 
Detection of acidic vesicular organelles (AVO) with acridine orange (AO) and acidic autophagic vacuoles with monodansylcadaverine $(M D C)$. T24 cells were seeded on sterile coverslips in tissue culture plates with a density of $5 \times 10^{4}$ cells/ per coverslip. After 0 or $200 \mu \mathrm{M}$ of baicalin treatment for $24 \mathrm{~h}$, cells were stained with either acridine orange (AO) or $0.1 \mathrm{mM}$ monodansylcadaverine (MDC) at $37^{\circ} \mathrm{C}$ for $10 \mathrm{~min}$. After three washes with PBS, cells were immediately visualized by fluorescence microscopy (Nikon, Melville, NY, USA) for the detection of acidic vesicular organelles and MDC-positive autophagic vacuoles $(18,47)$.

Western blot analysis. T24 cells (1x107/75-T flask) were treated with $0,50,100,150$ and $200 \mu \mathrm{M}$ of baicalin for $24 \mathrm{~h}$, then harvested, lysed and the total proteins were collected by SDS sample buffer. In brief, $\sim 30 \mu \mathrm{g}$ of protein from each treatment was resolved on $10 \%$ SDS-polyacrylamide gel electrophoresis (SDS-PAGE) and electro-transferred to a nitrocellulose membrane. The transferred membranes were blocked in $5 \%$ non-fat dry milk in $20 \mathrm{mM}$ Tris-buffered saline/ $0.05 \%$ Tween-20 for $1 \mathrm{~h}$ at room temperature followed by incubation with primary antibodies against indicated autophagic-associated proteins or AKT and autophagy pathway-related proteins at $4^{\circ} \mathrm{C}$ overnight. At the end of incubation, membranes were washed with Tris-buffered saline/Tween-20 and incubated with secondary antibodies conjugated with horseradish peroxidase (HRP). The blots were developed by a chemiluminescence kit (Millipore, Bedford, MA, USA), followed by X-ray film exposure. Each membrane was stripped and reprobed with anti- $\beta$-actin antibody to ensure equal protein loading during the experiment (18).

In vitro AKT kinase assay. In brief, T24 cells (1x107/75-T flask) were treated with $0,50,100,150$ and $200 \mu \mathrm{M}$ of baicalin for $6 \mathrm{~h}$. Cells were lysed in ice-cold lysis buffer provided by the kit. The $200 \mu \mathrm{g}$ of protein from each time-point of treatment was immuno-precipitated with $2 \mu \mathrm{g}$ of anti-AKT antibody overnight. Immuno-precipitates were extensively washed and then incubated with $1 \mu \mathrm{g}$ of GSK-3 $\alpha / \beta$ fusion protein substrate in $50 \mu \mathrm{l}$ of kinase buffer for $30 \mathrm{~min}$ at $30^{\circ} \mathrm{C}$. Reactions were stopped by SDS loading buffer and samples were separated on $12 \%$ SDS-PAGE. The phospho-GSK-3 $\alpha / \beta$ (Ser219) was detected by immunoblotting (18).

Statistical analysis. All the statistical results are presented as the mean \pm SEM for the indicated numbers of separate experiments. Statistical analyses of data were done using one-way ANOVA followed by Student's t-test and ${ }^{*} \mathrm{P}<0.05,{ }^{* *} \mathrm{P}<0.01$, ${ }^{* * * *} \mathrm{P}<0.001$ were considered statistically significant (18).

\section{Results}

Baicalin decreased the viability of T24 human bladder cancer cells. The human bladder cancer cells T24 were treated with baicalin $(0,50,100,150$ and $200 \mu \mathrm{M})$ for $24 \mathrm{~h}$. Results from the MTT assay showed that even though $50 \mu \mathrm{M}$ of baicalin did not reduce cell viability, increased concentrations of baicalin treatment $(100,150$ and $200 \mu \mathrm{M})$ significantly led to decrease of cell viability in T24 cells in a concentration-dependent manner (Fig. 2A). Fig. 2B showed that baicalin increased the
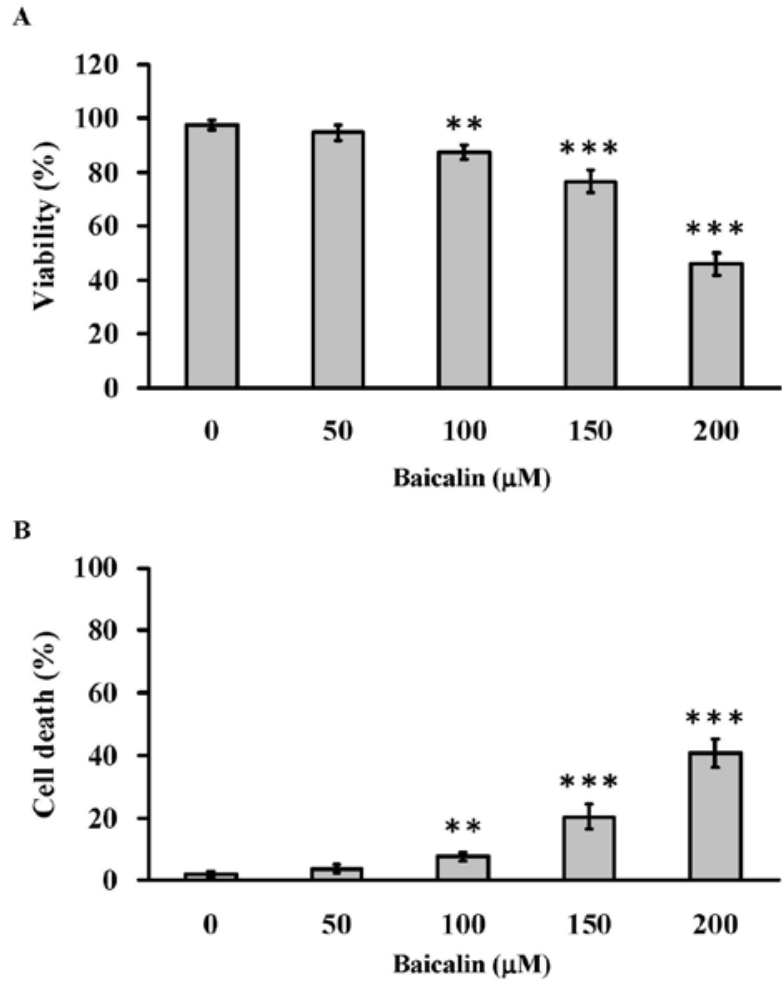

Figure 2. Effects of baicalin on cell viability and cell death in human bladder cancer T24 cells. (A) T24 cells were treated with various concentrations of baicalin $(0,50,100,150$ and $200 \mu \mathrm{M})$ for $24 \mathrm{~h}$. The viability of cells was determined by MTT assay. (B) The percentage of cell death was determined by the trypan blue exclusion assay. Data are expressed as the mean \pm SEM of three independent experiments. ${ }^{* *} \mathrm{p}<0.01,{ }^{* * * *} \mathrm{p}<0.001$, significant compared with the control $(0 \mu \mathrm{M})$ group.

number of cell death at 100,150 and $200 \mu \mathrm{M}$ in a concentration-dependent manner using the trypan blue exclusion assay.

Baicalin induces caspase-independent cell death in T24 cells. To verify whether baicalin induced apoptosis in T24 cells, cells were treated with baicalin $(0,50,100,150$ and $200 \mu \mathrm{M})$ for $24 \mathrm{~h}$ before subjected to TUNEL staining. Fig. $3 \mathrm{~A}$ indicated that the percentages of TUNEL-positive cells in baicalin-treated groups were $<5 \%$. In addition, to further examine whether the cell death caused by baicalin treatment was mediated through caspase-3 activation, protein samples collected from T24 cells after baicalin $(0,50,100,150$ and $200 \mu \mathrm{M})$ treatment were analyzed for caspase- 3 activity. The caspase- 3 activity assay showed no changes in baicalin-treated cells regardless of the baicalin concentrations (Fig. 3B). Our results demonstrated that apoptosis and the activation of caspase- 3 were not involved in baicalin-induced cell death.

Baicalin induces cell autophagy in T24 cells. We further investigated whether the cell death caused by baicalin treatment was mediated by autophagy. T24 cells were treated with baicalin $(0,50,100,150$ and $200 \mu \mathrm{M})$ for $24 \mathrm{~h}$ and the formation of autophagic vacuoles was examined under a phase contrast microscope. As shown in Fig. 4A, 100 and $200 \mu \mathrm{M}$ of baicalin treatment induced the formation of autophagic vacuoles, while baicalin at control and $50 \mu \mathrm{M}$ induced hardly 


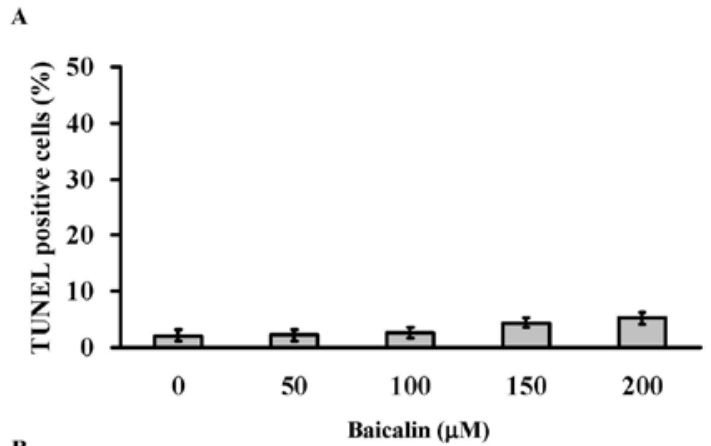

B

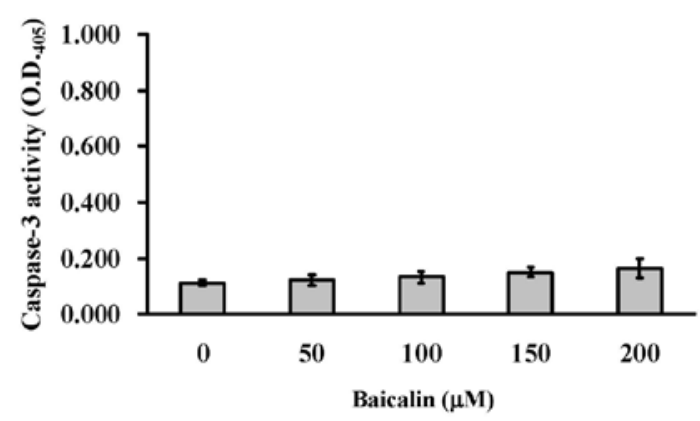

Figure 3. Effects of baicalin on cell apoptosis and caspase-3 activity in human bladder cancer T24 cells. (A) T24 cells were treated with various concentrations of baicalin $(0,50,100,150$ and $200 \mu \mathrm{M})$ for $24 \mathrm{~h}$. TUNEL staining was applied to determine the percentage of apoptotic cells (B) Analysis of caspase-3 activities in baicalin-treated T24 cells. After 24-h exposure to different doses of baicalin, the T24 cell lysate was pre-treated with z-DEVEfmk (the specific inhibitor of caspase-3) for $1 \mathrm{~h}$ followed by measuring the caspase- 3 activities. The $\mathrm{OD}_{405}$ of the released pNA was measured as the indication of caspase- 3 activities. Data are presented as the mean \pm SEM from three independent experiments.

$\mathbf{A}$
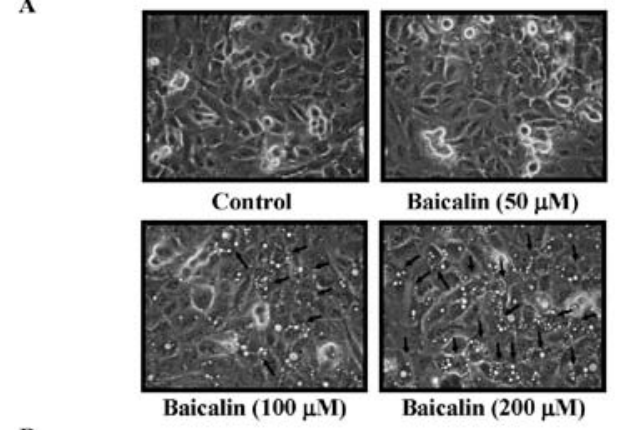

B

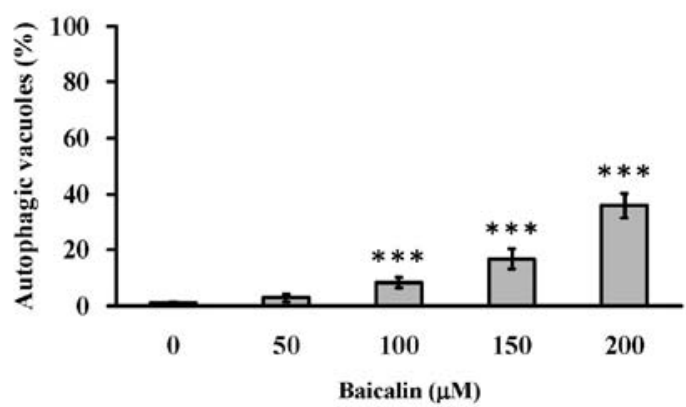

Figure 4. Baicalin induces cell autophagy in human bladder cancer T24 cells. (A) T24 cells were treated with baicalin $(0,50,100$ and $200 \mu \mathrm{M})$ for $24 \mathrm{~h}$ and were examined for the formation of autophagic vacuoles by a phase contrast microscope (magnification, x400). (B) The percentage of autophagic vacuoles from each treatment were quantified and presented as the mean \pm SEM. Data were collected from three independent experiments. ${ }^{* * *} \mathrm{p}<0.001$, significant compared with the control $(0 \mu \mathrm{M})$ group.
A

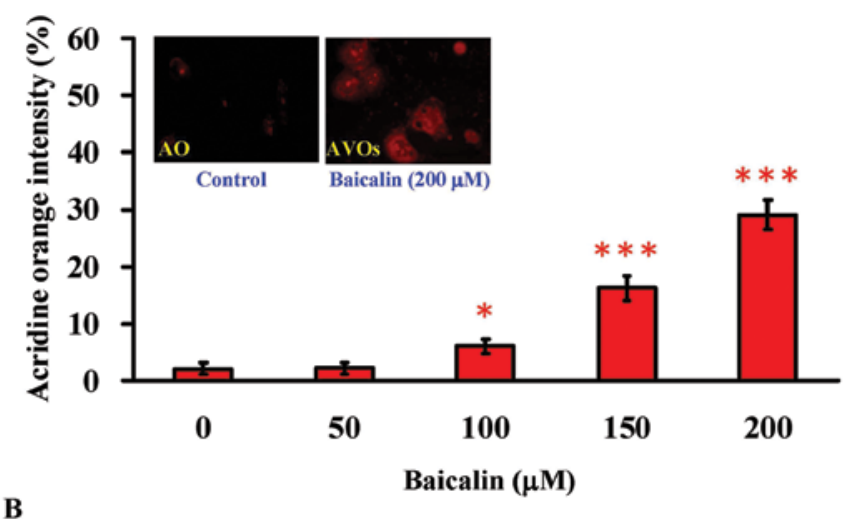

B

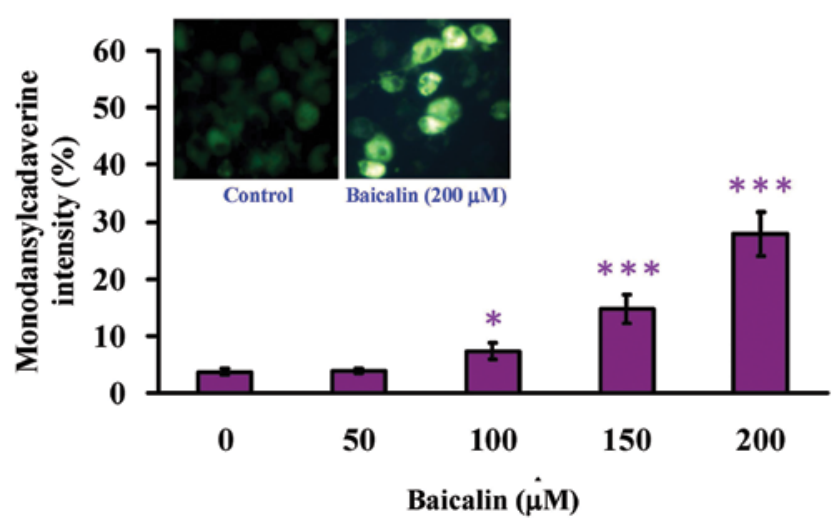

Figure 5. Baicalin induces autophagy markers in human bladder cancer T24 cells. (A) After treatment with various concentrations of baicalin $(0,50,100$, 150 and $200 \mu \mathrm{M}$ ) for $24 \mathrm{~h}$, the cytosolic acidic vesicular organelles (AVOs) were stained by acridine orange (AO) staining. The representative images of AO staining in the presence and absence of baicalin $(200 \mu \mathrm{M})$ in the upper panel; and quantitative data of AO intensity are shown. (B) Autophagic vacuoles in baicalin-treated T24 cells were stained by fluorescent probe monodansylcadaverine (MDC) in the upper panel and MDC intensities at each treatment are shown. ${ }^{*}<<0.05,{ }^{* * *} \mathrm{p}<0.001$, significant compared with the control $(0 \mu \mathrm{M})$ group.

any formation of autophagic vacuoles. In addition, the amount of autophagic vacuole formation was significantly elevated in a concentration-dependent manner in higher baicalin concentration groups $(\geq 100 \mu \mathrm{M})$ (Fig. 4B). Especially, upon the challenge of $200 \mu \mathrm{M}$ baicalin for $24 \mathrm{~h}, \sim 40 \%$ of cells manifested autophagic vacuoles.

One of the hallmarks of autophagic cell death is the cytosolic acidic vesicular organells (AVO) (48). Through the staining of acridine orange (AO), a lysotropic dye that emits bright red fluorescence inside the low $\mathrm{pH}$ acidic vesicles, AVO were noticeably observed in the cytoplasm of baicalin-treated T24 cells ( $200 \mu \mathrm{M}$ of baicalin) when compared to the control group by the fluorescence microscopy. In addition, as the concentration of baicalin increased, the measured AO intensity became stronger (Fig. 5A). Furthermore, we confirmed the autophagic cell death caused by baicalin treatment using monodansylcadaverine (MDC) staining. MDC is another widely used fluorescent marker that preferentially accumulates in autophagic vacuoles. As shown in Fig. 5B, T24 cells treated $200 \mu \mathrm{M}$ of baicalin for $24 \mathrm{~h}$ clearly showed autophagic vacuoles, while very few autophagic vacuoles were observed in the control group. Again, the MDC intensity increased as 
A

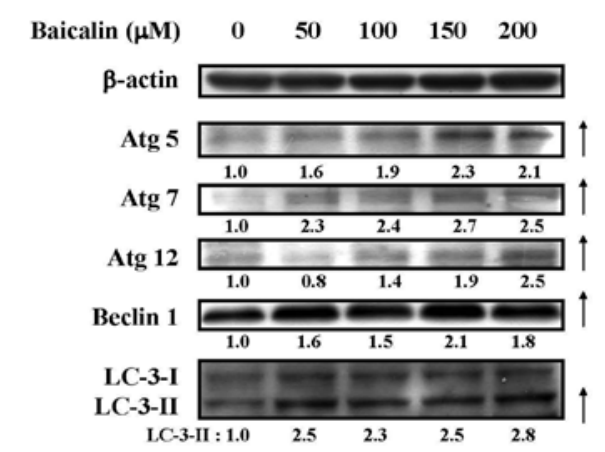

B

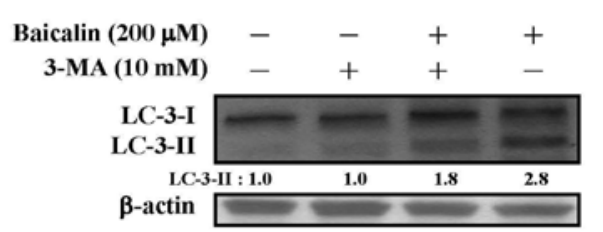

C

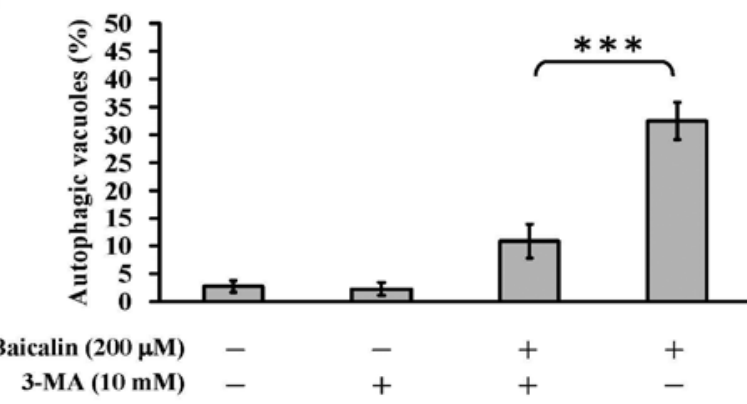

Figure 6. Baicalin increased the expression of autophage-associated proteins in human bladder cancer T24 cells. (A) T24 cells were treated with different concentrations $(0,50,100,150$ and $200 \mu \mathrm{M})$ of baicalin as indicated for $24 \mathrm{~h}$. The protein levels of Atg 5, Atg 7 and Atg 12, Beclin 1 and LC-3 were examined by western blot analysis. The numbers below each line of the protein bands indicate increasing folds of that protein level when compared to the control group (defined as 1.0). The protein level of $\beta$-actin was used as the internal standard. (B) Prior to baicalin treatment $(200 \mu \mathrm{M})$, cells were pre-treated with/without 3-MA $(10 \mathrm{mM})$ followed by western blot analysis of protein level of LC-3. (C) The percentage of autophagic vacuoles was measured after the exposure of T24 cells to baicalin and 3-MA interventions as indicated. Data are presented as the mean \pm SEM from three independent experiments. ${ }^{* * *} \mathrm{p}<0.001$, significant compared with the control $(0 \mu \mathrm{M})$ group.

the baicalin concentration increased. Our results indicated that autophagy was the mechanism underlying baicalin-induced cell death.

Baicalin regulates the autophagy-associated protein levels in T24 cells. It has been shown that the autophagic cell death is associated with the elevations of autophagosome formation protein levels. Those proteins includes light-chain-3 (LC-3), Atg complex (Atg 5, Atg 7 and Atg 12) and Beclin-1 $(18,49)$. As examined by western blot analysis, baicalin increased the protein expression of Atg 5, Atg 7 and Atg 12, Beclin-1 and LC-3 II (Fig. 6A). For example, when compared with the control group, the respective protein levels of Atg 5, Atg 7 and Atg 12, Beclin-1 and LC-3 II were 2.1-, 2.5-, 2.5-, 1.8and 2.8-fold higher after treated with $200 \mu \mathrm{M}$ of baicalin for $24 \mathrm{~h}$.

Among the afore-mentioned proteins, the microtubuleassociated protein light-chain 3 (LC-3) is a reliable autophagic
A

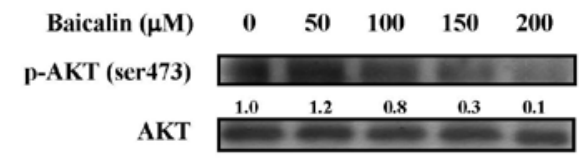

B

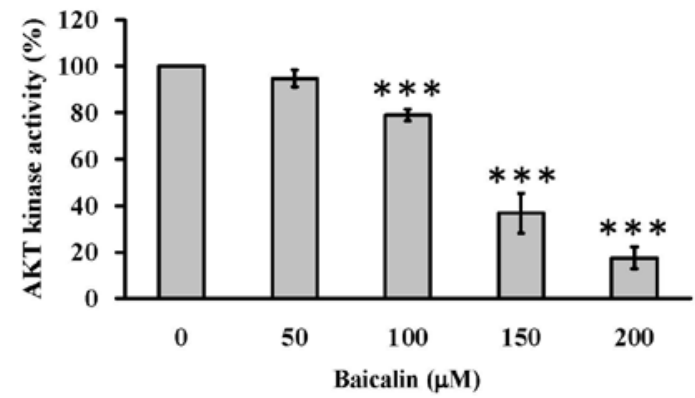

Figure 7. Baicalin inhibits AKT protein level and kinase activity in T24 human bladder cancer cells. (A) T24 cells were treated with different concentrations $(0,50,100,150$ and $200 \mu \mathrm{M})$ of baicalin as indicated for $12 \mathrm{~h}$. The protein levels of AKT and phosphor-AKT (ser 473) were examined by western blot analysis. (B) AKT kinase activity was measured by an in vitro kinase activity assay. Data are presented as the mean \pm SEM from three independent experiments. ${ }^{* * *} \mathrm{p}<0.001$, significant compared with the control $(0 \mu \mathrm{M})$ group.

membrane marker for the detection of early autophagosome formation $(50,51)$. The conversion of LC-3I to LC-3II is indicative of autophagic activity. We next examined whether 3-methyladenine (3-MA), a commonly used reagent that inhibits autophagy by blocking autophagosome formation via the inhibition of type III phosphatidylinositol 3-kinases (PI-3K), could attenuate the elevated LC-3 II expression induced by baicalin. As shown in Fig. 6B, $200 \mu \mathrm{M}$ of baicalin treatment upregulated the LC-3 II protein levels to 2.8-fold when compared to the control group. Nevertheless, 3-MA pretreatment $(10 \mathrm{mM})$ decreased the LC-3II expression level to 1.8 -fold in the presence of $200 \mu \mathrm{M}$ of baicalin. The quantitative data from the numbers of autophagic vacuoles also indicated that the baicalin-induced autophagic vacuoles formation was sharply diminished upon 3-MA pretreatment (Fig. 6C). The experimental results (Fig. 6) indicated that baicalin induced autophagic cell death through upregulation of proteins associated with autophagosome formation and it could be attenuated by 3-MA.

Baicalin blocks the AKT signaling in T24 cells. The AKT activity has been demonstrated to contribute to autophagic cell death $(6,40)$. We next performed western blot analysis and AKT kinase activity assay to investigate whether the AKT signaling was involved in the baicalin-induced autophagic cell death in T24 cells. The present study showed that baicalin decreased the phosphor-AKT (Ser473) protein levels in T24 cells in a concentration-dependent manner (Fig. 7A). In addition, baicalin inhibited AKT kinase activity and the inhibition was concentration-dependent (Fig. 7B). Our data implied that baicalin induced cell autophagy in T24 cells through blocking the AKT signaling. 


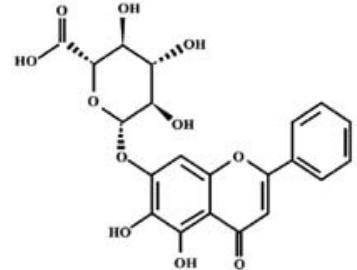

Baicalin

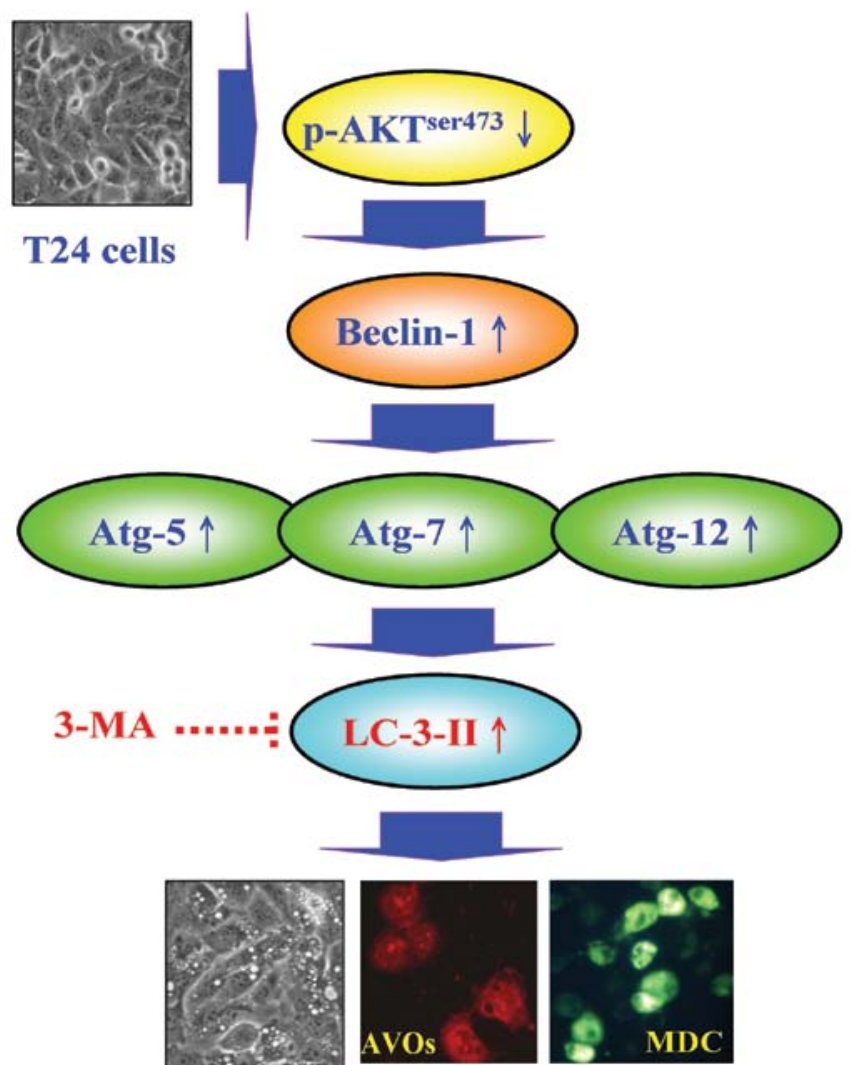

Autophagic vacuoles

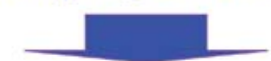

Autophagy

Figure 8. The proposed model of baicalin induced cell autophagy in T24 human bladder cancer cells.

\section{Discussion}

Previous studies have showed that Scutellaria baicalensis Gerogi containing over 30 different kinds of flavonoids $(24,52,53)$, including baicalin, baicalein, oroxylin A and wogonin (53-55). It was reported that ethanol extracts of Scutellaria baicalensis Gerogi prevent oxidative damage $(56,57)$ and has anti-inflammation $(58,59)$ and anti-angiogenesis effects (60). In addition, Scutellaria baicalensis Gerogi extract triggers $\mathrm{G}_{2} / \mathrm{M}$ arrest and caspase-dependent apoptosis by modulating ERK pathway in HSC-T6 cells (58). In the present study, we focused on the baicalin from Scutellaria baicalensis Gerogi for their anticancer effect on human bladder cancer T24 cells. Baicalin is a natural flavonoids compound with anticancer activity and low toxicity against normal cells $(61,62)$. Previous reports showed that baicalin exerted anti-proliferative ability and induced apoptotic effects in many cancer cell lines (CA46, SW620, HCT-116, HL-60/ ADR and HL-60) $(40,41,43,44,63,64)$. In this study, we investigated the anticancer effects of baicalin on T24 human bladder cancer cells in vitro. Our results showed that baicalin exerted a significant anti-proliferative effect on T24 cells (Fig. 2A). Baicalin is a new anticancer agent and has apoptotic effect on
T24 cells. However, the apoptotic TUNEL-positive cells and caspase-3 activity did not change in baicalin-treated T24 cells (Fig. 3). Data suggested that there may be another mechanism involved in baicalin-induced cell death in T24 cells.

Many studies have suggested the autophagy has a cancer suppressor role (65). Several traditional Chinese medicines such as arsenic trioxide $\left(\mathrm{AS}_{2} \mathrm{O}_{3}\right)(66)$, berberine (67), bufalin (18) and kaempferol (68) have been demonstrated to induce autophagy and to exert anticancer activity in cancer cells. Intriguingly, baicalin-induced autophagy in T24 cells was demonstrated by autophagic vesicle formation (Fig. 4). Baicalin induced autophagy generation shown by larger bright-red $\mathrm{AO}$-stained vacuoles (Fig. 5A) and induction of the LC3 cleavage (Fig. 6A). In contrast, protein levels of the LC3-II, Beclin-1, Atg 5, Atg 7 and Atg 12 were upregulated in T24 cells after baicalin treatment (Fig. 6A). When T24 cells were pre-treated with 3-MA followed by treatment with baicalin, LC3 protein cleavage (Fig. 6B) and autophagic vesicle formation (Fig. 6C) were significantly decreased compared with the baicalin alone treatment group. Our results demonstrated that baicalin-induced cell death possibly involved autophagy, and is the first detailed evidence that baicalin induced autophagy in T24 cells. Our 
findings are in agreement with previous studies that baicalin induced autophagy in SMMC-7721 cells (45).

Akt serine/threonine kinase [also called protein kinase B (PKB)] is one of the most regularly activated protein kinases in human bladder cancer (69-71). Activation of Akt is associated with anti-apoptosis, cell proliferation and cellular energy metabolism (72). The AKT pathway is frequently activated in human bladder cancer cells. Askham et al demonstrated that the AKT1 G49A (E17K) mutation led to constitutive AKT1 activation and was found in $4.8 \%$ bladder cancer cell lines and $2.7 \%$ bladder tumors (73). Regulating the Akt pathway is potentially essential for developing therapeutic inhibitors in human bladder cancer. Dickstein et al demonstrated that the AKT inhibitor AZ7328 has synergistic effect on inducing apoptosis with autophagy inhibitors in human bladder cancer cells (74). Wu et al demonstrated that PI-3 kinase inhibitor LY294002 inhibits cell proliferation and sensitizes doxorubicin in human bladder cancer cells (75). Our study demonstrated baicalin induced autophagy accompanied with downregulation of phospho-AKT (Ser473) protein level (Fig. 7A) and Akt kinase activity (Fig. 7B). Previous studies demonstrated that baicalin induced apoptotic cell death through inhibiting the AKT signaling pathway in CA46 Burkitt lymphoma and leukemia HL-60/ADR cells (40,76). In the present study, the result showed that the AKT pathway is associated with the induction of autophagy in baicalin-treated T24 cells.

The molecular mechanisms underlying the inhibitory effect of baicalin on T24 cell proliferation are summarized in Fig. 8. In conclusion, baicalin induces autophagy through the Akt signaling pathway in T24 human bladder cancer cells. Our findings imply that baicalin may be used as a novel anticancer drug candidate for the treatment of human bladder cancer.

\section{Acknowledgements}

We thank the grant-in-aid DOH101-TD-C-111-005 from Taiwan Department of Health, China Medical University Hospital Cancer Research Center of Excellence. This study was supported by the grant from the National Science Council, Republic of China (Taiwan). This study was also supported in part by grant from China Medical University (CMU101-S-27) awarded to J.-S. Yang.

\section{References}

1. Golstein P and Kroemer G: Cell death by necrosis: towards a molecular definition. Trends Biochem Sci 32: 37-43, 2007.

2. Bustamante-Marin X, Quiroga C, Lavandero S, Reyes JG and Moreno RD: Apoptosis, necrosis and autophagy are influenced by metabolic energy sources in cultured rat spermatocytes. Apoptosis 17: 539-550, 2012.

3. Edinger AL and Thompson CB: Death by design: apoptosis, necrosis and autophagy. Curr Opin Cell Biol 16: 663-669, 2004.

4. Mah LY and Ryan KM: Autophagy and cancer. Cold Spring Harb Perspect Biol 4: a008821, 2012.

5. Lockshin RA and Zakeri Z: Cell death in health and disease. J Cell Mol Med 11: 1214-1224, 2007.

6. Glick D, Barth S and Macleod KF: Autophagy: cellular and molecular mechanisms. J Pathol 221: 3-12, 2010.

7. Kaminskyy V and Zhivotovsky B: Proteases in autophagy. Biochim Biophys Acta 1824: 44-50, 2012.

8. Chen Y and Yu L: Autophagic lysosome reformation. Exp Cell Res 19: 142-146, 2013.

9. Mizushima N: The role of the Atg1/ULK1 complex in autophagy regulation. Curr Opin Cell Biol 22: 132-139, 2010.
10. Reggiori F: 1. Membrane origin for autophagy. Curr Top Dev Biol 74: 1-30, 2006.

11. Tanida I, Ueno T and Kominami E: LC3 conjugation system in mammalian autophagy. Int J Biochem Cell Biol 36: 2503-2518, 2004.

12. Yang WL, Perillo W, Liou D, Marambaud P and Wang P: AMPK inhibitor compound $\mathrm{C}$ suppresses cell proliferation by induction of apoptosis and autophagy in human colorectal cancer cells. J Surg Oncol 106: 680-688, 2012.

13. Ge W, Guo R and Ren J: AMP-dependent kinase and autophagic flux are involved in aldehyde dehydrogenase-2-induced protection against cardiac toxicity of ethanol. Free Radic Biol Med 51: 1736-1748, 2011

14. Wu Y, Li X, Zhu JX, et al: Resveratrol-activated AMPK/SIRT1/ autophagy in cellular models of Parkinson's disease. Neurosignals 19: 163-174, 2011.

15. Li ZY, Yang Y, Ming M and Liu B: Mitochondrial ROS generation for regulation of autophagic pathways in cancer. Biochem Biophys Res Commun 414: 5-8, 2011.

16. Dagda RK, Zhu J, Kulich SM and Chu CT: Mitochondrially localized ERK2 regulates mitophagy and autophagic cell stress: implications for Parkinson's disease. Autophagy 4: 770-782, 2008.

17. Nishiyama Y, Shimada Y, Yokoi T, et al: Akt inactivation induces endoplasmic reticulum stress-independent autophagy in fibroblasts from patients with Pompe disease. Mol Genet Metab 107: 490-495 2012.

18. Tsai SC, Yang JS, Peng SF, et al: Bufalin increases sensitivity to AKT/mTOR-induced autophagic cell death in SK-HEP-1 human hepatocellular carcinoma cells. Int J Oncol 41: 1431-1442 2012.

19. Chen J, Crawford R and Xiao Y: Vertical inhibition of the PI3K/ $\mathrm{Akt} / \mathrm{mTOR}$ pathway for the treatment of osteoarthritis. J Cell Biochem: Aug 28, 2012 (Epub ahead of print). doi: 10.1002/ jcb.24362.

20. Zeng T, Zhang CL, Song FY, et al: PI3K/Akt pathway activation was involved in acute ethanol-induced fatty liver in mice. Toxicology 296: 56-66, 2012.

21. Martelli AM, Evangelisti C, Follo MY, et al: Targeting the phosphatidylinositol 3-kinase/Akt/mammalian target of rapamycin signaling network in cancer stem cells. Curr Med Chem 18: 2715-2726, 2011

22. Hugel HM, Jackson N, May BH and Xue CC: Chinese herbs for dementia diseases. Mini Rev Med Chem 12: 371-379, 2012.

23. Chen $\mathrm{S}$, Wu T, Kong $X$ and Yuan $\mathrm{H}$ : Chinese medicinal herbs for measles. Cochrane Database Syst Rev: Nov 9, 2011, CD005531. doi: 10.1002/14651858.CD005531.pub4.

24. Yuan Y, Shuai L, Chen S, Huang L, Qin S and Yang Z: Flavonoids and antioxidative enzymes in temperature-challenged roots of Scutellaria baicalensis Georgi. Z Naturforsch C 67: 77-85, 2012.

25. Ma AT, Zhong XH, Liu ZM, et al: Protective effects of baicalin against bromocriptine induced abortion in mice. Am J Chin Med 37: 85-95, 2009.

26. Waisundara VY, Siu SY, Hsu A, Huang D and Tan BK: Baicalin upregulates the genetic expression of antioxidant enzymes in Type-2 diabetic Goto-Kakizaki rats. Life Sci 88: 1016-1025, 2011.

27. Bing-Rong Z, Song-Liang J, Xiao EC, et al: Protective effect of the Baicalin against DNA damage induced by ultraviolet $\mathrm{B}$ irradiation to mouse epidermis. Photodermatol Photoimmunol Photomed 24: 175-182, 2008.

28. Li HY, Hu J, Zhao S, et al: Comparative study of the effect of baicalin and its natural analogs on neurons with oxygen and glucose deprivation involving innate immune reaction of TLR2/ TNFalpha. J Biomed Biotechnol: Mar 21, 2012 (Epub). 267890, doi: $10.1155 / 2012 / 267890$.

29. Cao Y, Mao X, Sun C, et al: Baicalin attenuates global cerebral ischemia/reperfusion injury in gerbils via anti-oxidative and anti-apoptotic pathways. Brain Res Bull 85: 396-402, 2011.

30. de Carvalho RS, Duarte FS and de Lima TC: Involvement of GABAergic non-benzodiazepine sites in the anxiolytic-like and sedative effects of the flavonoid baicalein in mice. Behav Brain Res 221: 75-82, 2011.

31. Zhu J, Wang J, Sheng Y, et al: Baicalin improves survival in a murine model of polymicrobial sepsis via suppressing inflammatory response and lymphocyte apoptosis. PLoS One 7: e35523, 2012.

32. Fu S, Sun C, Tao X and Ren Y: Anti-inflammatory effects of active constituents extracted from Chinese medicinal herbs against Propionibacterium acnes. Nat Prod Res 26: 1746-1749, 2012. 
33. Chu ZY, Chu $M$ and Teng Y: Effect of baicalin on in vivo anti-virus. Zhongguo Zhong Yao Za Zhi 32: 2413-2415, 2007 (In Chinese).

34. Kitamura K, Honda M, Yoshizaki H, et al: Baicalin, an inhibitor of HIV-1 production in vitro. Antiviral Res 37: 131-140, 1998.

35. Qiao H, Han H, Hong D, Ren Z, Chen Y and Zhou C: Protective effects of baicalin on carbon tetrachloride induced liver injury by activating PPARgamma and inhibiting TGFbeta1. Pharm Biol 49: 38-45, 2011.

36. Hwang JM, Wang CJ, Chou FP, et al: Protective effect of baicalin on tert-butyl hydroperoxide-induced rat hepatotoxicity. Arch Toxicol 79: 102-109, 2005.

37. Chiu YW, Lin TH, Huang WS, et al: Baicalein inhibits the migration and invasive properties of human hepatoma cells. Toxicol Appl Pharmacol 255: 316-326, 2011.

38. Shieh DE, Cheng HY, Yen MH, Chiang LC and Lin CC: Baicalin-induced apoptosis is mediated by Bcl-2-dependent, but not p53-dependent, pathway in human leukemia cell lines. Am J Chin Med 34: 245-261, 2006

39. Motoo Y and Sawabu N: Antitumor effects of saikosaponins, baicalin and baicalein on human hepatoma cell lines. Cancer Lett 86: 91-95, 1994.

40. Huang Y, Hu J, Zheng J, et al: Down-regulation of the PI3K/Akt signaling pathway and induction of apoptosis in CA46 Burkitt lymphoma cells by baicalin. J Exp Clin Cancer Res 31: 48, 2012

41. Chen WC, Kuo TH, Tzeng YS and Tsai YC: Baicalin induces apoptosis in SW620 human colorectal carcinoma cells in vitro and suppresses tumor growth in vivo. Molecules 17: 3844-3857, 2012.

42. Lee DH, Kim C, Zhang L and Lee YJ: Role of p53, PUMA, and Bax in wogonin-induced apoptosis in human cancer cells. Biochem Pharmacol 75: 2020-2033, 2008.

43. Zheng J, Hu JD, Chen YY, et al: Baicalin induces apoptosis in leukemia HL-60/ADR cells via possible down-regulation of the PI3K/Akt signaling pathway. Asian Pac J Cancer Prev 13: 1119-1124, 2012

44. Lu HF, Hsueh SC, Ho YT, et al: ROS mediates baicalin-induced apoptosis in human promyelocytic leukemia HL-60 cells through the expression of the Gadd153 and mitochondrial-dependent pathway. Anticancer Res 27: 117-125, 2007.

45. Zhang X, Tang X, Liu H, Li L, Hou Q and Gao J: Autophagy induced by baicalin involves downregulation of CD147 in SMMC-7721 cells in vitro. Oncol Rep 27: 1128-1134, 2012.

46. Huang WW, Yang JS, Pai SJ, et al: Bufalin induces $\mathrm{G}(0) / \mathrm{G}(1)$ phase arrest through inhibiting the levels of cyclin D, cyclin E, CDK2 and CDK4, and triggers apoptosis via mitochondrial signaling pathway in T24 human bladder cancer cells. Mutat Res 732: 26-33, 2012 .

47. Kim JY, Cho TJ, Woo BH, et al: Curcumin-induced autophagy contributes to the decreased survival of oral cancer cells. Arch Oral Biol 57: 1018-1025, 2012.

48. Munafo DB and Colombo MI: A novel assay to study autophagy: regulation of autophagosome vacuole size by amino acid deprivation. J Cell Sci 114: 3619-3629, 2001.

49. McCoy F, Hurwitz J, McTavish N, et al: Obatoclax induces Atg7dependent autophagy independent of beclin-1 and BAX/BAK Cell Death Dis 1: e108, 2010.

50. Barth S, Glick D and Macleod KF: Autophagy: assays and artifacts. J Pathol 221: 117-124, 2010.

51. Mizushima N and Yoshimori T: How to interpret LC3 immunoblotting. Autophagy 3: 542-545, 2007.

52. Yu K, Gong Y, Lin Z and Cheng Y: Quantitative analysis and chromatographic fingerprinting for the quality evaluation of Scutellaria baicalensis Georgi using capillary electrophoresis. J Pharm Biomed Anal 43: 540-548, 2007.

53. Zhou XQ, Liang H, Lu XH, Cai SQ, Wang B and Zhao YY: Flavonoids from Scutellaria baicalensis and their bioactivities. Beijing Da Xue Xue Bao 41: 578-584, 2009 (In Chinese).

54. Liu B, Shi RB and Zhu LJ: HPLC fingerprint of flavonoids of Kushen Tang and its correlation to Scutellaria baicalensis and Sophora flavescens. Zhongguo Zhong Yao Za Zhi 32: 1631-1634, 2007 (In Chinese).

55. Kim YH, Jeong DW, Kim YC, Sohn DH, Park ES and Lee HS: Pharmacokinetics of baicalein, baicalin and wogonin after oral administration of a standardized extract of Scutellaria baicalensis, PF-2405 in rats. Arch Pharm Res 30: 260-265, 2007.
56. Hirunuma M, Shoyama Y, Sasaki K, et al: Flavone-catalyzed apoptosis in Scutellaria baicalensis. Phytochemistry 72: 752-760, 2011.

57. Choi J, Conrad CC, Malakowsky CA, Talent JM, Yuan CS and Gracy RW: Flavones from Scutellaria baicalensis Georgi attenuate apoptosis and protein oxidation in neuronal cell lines. Biochim Biophys Acta 1571: 201-210, 2002

58. Pan TL, Wang PW, Leu YL, Wu TH and Wu TS: Inhibitory effects of Scutellaria baicalensis extract on hepatic stellate cells through inducing G2/M cell cycle arrest and activating ERK-dependent apoptosis via Bax and caspase pathway. J Ethnopharmacol 139: 829-837, 2012.

59. Li HB, Jiang Y and Chen F: Separation methods used for Scutellaria baicalensis active components. J Chromatogr B Analyt Technol Biomed Life Sci 812: 277-290, 2004.

60. Wang S, Zheng Z, Weng Y, et al: Angiogenesis and anti-angiogenesis activity of Chinese medicinal herbal extracts. Life Sci 74: 2467-2478, 2004

61. Zhu H, Wang Z, Xing Y, et al: Baicalin reduces the permeability of the blood-brain barrier during hypoxia in vitro by increasing the expression of tight junction proteins in brain microvascular endothelial cells. J Ethnopharmacol 141: 714-720, 2012.

62. Hu Q, Noor M, Wong YF, et al: In vitro anti-fibrotic activities of herbal compounds and herbs. Nephrol Dial Transplant 24: 3033-3041, 2009.

63. Yang BL, Chen HJ, Chen YG, et al: Inhibitory effects of baicalin on orthotopic xenografts of colorectal cancer cells that are deficient in a mismatch repair gene in nude mice. Int J Colorectal Dis: Aug 23, 2012 (Epub ahead of print).

64. Ren X, Li CL, Wang HX, et al: Molecular mechanism of HL-60 cell apoptosis induced by baicalin. Zhongguo Shi Yan Xue Ye Xue Za Zhi 20: 847-851, 2012 (In Chinese).

65. Carew JS, Kelly KR and Nawrocki ST: Autophagy as a target for cancer therapy: new developments. Cancer Manag Res 4: 357-365, 2012.

66. Goussetis DJ, Altman JK, Glaser H, McNeer JL, Tallman MS and Platanias LC: Autophagy is a critical mechanism for the induction of the antileukemic effects of arsenic trioxide. J Biol Chem 285: 29989-29997, 2010.

67. Wang N, Feng Y, Zhu M, et al: Berberine induces autophagic cell death and mitochondrial apoptosis in liver cancer cells: the cellular mechanism. J Cell Biochem 111: 1426-1436, 2010.

68. Filomeni G, Desideri E, Cardaci S, et al: Carcinoma cells activate AMP-activated protein kinase-dependent autophagy as survival response to kaempferol-mediated energetic impairment. Autophagy 6: 202-216, 2010.

69. Shahjee HM, Koch KR, Guo L, Zhang CO and Keay SK: Antiproliferative factor decreases Akt phosphorylation and alters gene expression via CKAP4 in T24 bladder carcinoma cells. J Exp Clin Cancer Res 29: 160, 2010.

70. Chen M, Cassidy A, Gu J, et al: Genetic variations in PI3K-AKTmTOR pathway and bladder cancer risk. Carcinogenesis 30: 2047-2052, 2009.

71. Champelovier P, El Atifi M, Mantel F, et al: In vitro tumoral progression of human bladder carcinoma: role for TGFbeta. Eur Urol 48: 846-851, 2005.

72. Stueckle TA, Lu Y, Davis ME, et al: Chronic occupational exposure to arsenic induces carcinogenic gene signaling networks and neoplastic transformation in human lung epithelial cells. Toxicol Appl Pharmacol 261: 204-216, 2012.

73. Askham JM, Platt F, Chambers PA, Snowden H, Taylor CF and Knowles MA: AKT1 mutations in bladder cancer: identification of a novel oncogenic mutation that can co-operate with E17K. Oncogene 29: 150-155, 2010.

74. Dickstein RJ, Nitti G, Dinney CP, Davies BR, Kamat AM and McConkey DJ: Autophagy limits the cytotoxic effects of the AKT inhibitor AZ7328 in human bladder cancer cells. Cancer Biol Ther 13: 1325-1338, 2012.

75. Wu D, Tao J, Xu B, et al: Phosphatidylinositol 3-kinase inhibitor LY294002 suppresses proliferation and sensitizes doxorubicin chemotherapy in bladder cancer cells. Urol Int 86: 346-354, 2011.

76. Zheng J, Hu JD, Huang Y and Chen BY: Effects of baicalin on proliferation and apoptosis of adriamycin-resistant human leukemia HL-60/ADR cells. Zhongguo Shi Yan Xue Ye Xue Za Zhi 17: 1198-1202, 2009 (In Chinese). 